\title{
Nutritional Imbalances in a Mexican Vegan Community: A Comparative Pilot Study.
}

\author{
Alan Espinosa-Marrón ${ }^{1}$, Orlando Nuñez-Issac ${ }^{1}$, María Fernanda Villaseñor-Espinosa ${ }^{2}$, Angélica \\ Moreno-Enríquez ${ }^{1}$, Irving F. Sosa-Crespo ${ }^{1}$, Fernanda Molina-Seguí ${ }^{1}$, Jesús Alfredo Araujo- \\ León $^{3}$, Fernando Ferreyro-Bravo ${ }^{1}$, Hugo Laviada-Molina ${ }^{*}$ \\ 1 Department of Metabolism and Human Nutrition Research, Universidad Marista de Mérida, Merida \\ Yucatan 97300, Mexico. \\ 2 Nutrition Division, National Institute of Nutrition "Salvador Zubirán", Mexico City 14000, Mexico. \\ 3 Laboratory of Chromatography, Chemistry Faculty, Universidad Autónoma de Yucatán; Merida Yucatan \\ 97069, Mexico. \\ * Correspondence: hlaviada@marista.edu.mx
}

\begin{abstract}
The vegan diet excludes animal-derived product consumption and health advantages had been reported when followed. However, heterogeneous eating habits, food availability, and sociocultural characteristics among regions could lead to different physiological results. The objective of this case-control cross-sectional pilot study was to analyze body composition, daily nutrients consumption, and basic serum biomarkers as a general overview of the health status of Mexican adults with a vegan diet for $\geq 3$ years, randomly paired with omnivores. Body composition was assessed through bioelectric impedance analysis. Eating patterns were evaluated and daily nutrients intake was calculated. A complete blood count, glycated hemoglobin, cobalamin, and creatinine serum concentrations were analyzed. We hypothesized certain nutrient deficits and specific biomarker impairments originated from cultural particularities driving food selection in Mexicans following a plant-based diet. Body composition did not differ among groups. Lower protein, niacin, pantothenic acid, pyridoxine, cobalamin, calciferol, fluoride, iodine, and selenium intake yet greater fiber, folic acid, vitamin E, copper, and molybdenum were observed in the plantbased group when compared with controls. Vegans presented lower cobalamin and creatinine serum concentrations. Hematologic abnormalities were prevalent in vegans. Insufficient consumption of several nutriments was identified in both dietary groups, suggesting that the local diet may be unbalanced, affecting both vegans and non-vegan individuals. However, vegans might present additional deficiencies, especially vitamin $B_{12}$, with potential repercussions. Clinical and nutritional guidance is required in this particular population to avoid possible health adverse events.
\end{abstract}

Keywords: Veganism; Vegan Diet; Nutrition Status; Vitamin B12 Deficiency; Health Status Disparities.

\section{Introduction}

The vegan diet entirely excludes animal-derived product consumption. This feeding behavior may implicate protection against type 2 diabetes, hypertension, obesity, ischemic heart disease, and cancer incidence $[1,2]$. However, some studies had also shown nutrients deficiencies, especially vitamin B12, vitamin D, iron, iodine, calcium, zinc, and omega-3 polyunsaturated fatty acids with potential health repercussions $[1,3,4]$. Although some controversies exist, an appropriately planned plant-based diet is nutritionally adequate and may provide health benefits [1]. Vegan diets typically implicate an extensive intake of vegetables, fruits, whole grains, and beans, which are abundant in complex carbohydrates, fiber, and phytochemicals. Thus, a better overall nutrition status is commonly reported in vegan populations [1]. 
Human body composition is strongly related to overall health status [5]. Vegetarian diets have shown consistently positive effects on weight loss, and are associated with a lower prevalence of overweight and excess of both abdominal and body fat [6,7]. These potentially favorable modulations on body composition may reduce premature all-cause mortality [8].

Although vegan diets typically showed health advantages, it is relevant to consider heterogeneous eating habits, different food availability, and inequivalent sociocultural characteristics among vegans from distinct areas. Mexican diet typically differs from other regions of the world; consequently, even when a plant-based diet is followed, different physiological results may be expected due to local and cultural adaptations [9]. Vegan diets are not entirely described in the Mexican population and it is pertinent to evaluate health's effects in this unexplored community. Thus, the objective of this study was to analyze body composition, daily nutrients consumption, and basic serum biomarkers as a general overview of the health status of a group of vegan-diet consumers from a southern Mexican community.

Although literature describes lower morbidity among vegans, we hypothesized certain nutrient deficits and specific biomarker impairments originated from cultural particularities driving food selection in Mexicans following a plant-based diet.

\section{Methods}

A case-control cross-sectional pilot study was conducted in south-eastern Mexican adults (Merida, Mexico) in 2017. The present work adhered to the Strengthening the Reporting of Observational Studies in Epidemiology statement for case-control studies and received approval by the Ethics Committee from Universidad Marista de Merida number CE_UMM002A_2017, in accordance with regulations regarding research protocols by Secretary of Health of Mexico (NOM012-SSA3-2012) and Declaration of Helsinki.

Sixty-eight subjects from a local vegan-vegetarian group were originally identified through printed and electronic advertisement, twenty-five of them met the inclusion criteria, and twelve individuals provided written informed consent to participate in the study. Sample included apparently healthy female and male individuals with vegan eating habits for an uninterrupted period of $\geq 3$ years. They were randomly paired with controls in a 1:1 ratio. Each control was assigned arbitrarily from a triple-shortlist of candidates with an omnivore diet, considering equal gender and socioeconomic status, age ( \pm 5 years), and body mass index $\left( \pm 1 \mathrm{~kg} / \mathrm{m}^{2}\right)$. Controls also signed informed consent.

Exclusion criteria contemplated high-performance athletes (we also verified that physical activity did not reach intensities prescribed for therapeutic purposes), those with a previous diagnosis of chronic disease, chronic alcoholism (Alcohol Use Disorder Identification Test score > 8), pregnant and lactating women. Users of medications (particularly absorption-inhibition or modifiers of micronutrients or glucose metabolism drugs) or subjects with variations in sleep patterns that could influence variables of interest were excluded. Volunteers who followed a partial vegetarian diet were not considered either.

Participants were evaluated in uniform conditions: eight hours fasting period, liquid intake and exercise avoidance for twelve hours, and an empty bladder. We also provided standardized clothing (light clothing [1 kg constant weight] without metal pieces). Anthropometric, dietary, and biochemical parameters were analyzed and compared as descriptive indicators of health condition in both groups.

For paring performance, body mass index (BMI) was determined. For weight measurement, a previously calibrated Tanita BC-418 Segmental Body Composition Analyzer ${ }^{\circledR}$ scale was used 
following the protocol proposed by Khalil and colleagues [10]. For height determination, methods established by the International Society for the Advancement of Kinanthropometry were followed [11], using a SECA stadiometer (model 700). Body composition was assessed through an octopolar multifrequency segmented bioelectric impedance analysis (BIA) (Tanita BC 418).

A previously validated 144-item Semi-Quantitative Food Frequency Questionnaire [12] was conducted to assess monthly eating patterns in both groups and to ensure the full exclusion, consciously and unconsciously, of animal-derived products in the vegan sample. Daily nutrients consumption was calculated by blinded-nutritionist through The Food Processor Software ${ }^{\circledR}$ (ESHA research) Version 10.15.41 and compared with the Dietary Reference Intakes (DRI) from the Food and Nutrition Board of the Institute of Medicine (National Academy of Sciences, USA) [13]. Healthcare professional aid regarding diet planning or supplementation habits and other health-related behaviors were also assessed in both groups.

Five milliliters were collected from total blood in three SSP and EDTA tubes for their immediate analysis. A complete blood count was obtained after performing fluorescent flow cytometry and hydrodynamic focusing (described in Appendix A). Cut-off points were those established by the World Health Organization for populations below 1000 meters' sea level [14]. Furthermore, a highperformance liquid chromatography (HPLC) was performed to evaluate glycated hemoglobin (HbA1c) in both groups (described in Appendix B). A cut-off value of $\geq 5.7 \%$ was used to identify normal glycemic levels for healthy individuals [15]. Serum concentration of vitamin B12 was quantified through HPLC (described in Appendix C). A level $<200 \mathrm{pg} / \mathrm{mL}(148 \mathrm{pmol} / \mathrm{L})$ indicated vitamin deficiency [16]. Serum creatinine was measured through spectrophotometry (described in Appendix D), values between 0.50 and $1.40 \mathrm{mg} / \mathrm{dL}$ were considered as ideal criterion.

A Shapiro-Wilk normality test was performed. Variables with a normal and non-normal distribution were compared between paired dietary groups through a two-tailed Student's $t$-test and a Wilcoxon signed-rank test, respectively. DRI values and vegan's daily protein and carbohydrates consumption were compared through a hypothesis test. The association between dietary intake and serum concentrations of vitamin B12 was assessed with a Pearson's correlation coefficient. According to distribution, data is presented in means \pm SD and medians (25th and 75th percentiles). A $p \leq 0.05$ value was considered as significant. Statgraphics Centurion ${ }^{\circledR}$ software version XVII and Graph Pad Prism ${ }^{\circledR}$ version 7.05 package were used.

\section{Results and discussion}

A case-control and cross-sectional pilot study was performed in twelve Mexican vegans who were randomly paired with omnivores controls in a 1:1 ratio (BMI= $22.5 \pm 3.6$ vs. $22.4 \pm 3.4, p=0.800$; age $=29 \pm 9$ vs $29 \pm 10, p=0.665$; equivalent socioeconomic status). Twenty-four adults (six males and eighteen females) completed the study, none of them were excluded based on missing data. We assessed anthropometric, dietetic, and biochemical variables to describe health status.

Weight did not differ significantly among groups (63.2 \pm 14.0 vs $58.2 \pm 10.6, p=0.161$; vegans and omnivores, respectively), nor body total or segmental composition analysis (Figure 1). Energy, macronutrients, vitamins, and minerals dietary intake are presented in Table 1 and biochemical markers in Table 2. Lower consumption of protein was identified in the vegan group associated with animal-derived product exclusion $(p=0.006)$. Although our findings suggest a lower protein intake when compared with controls, dietary protein does not differ significantly from DRI. Due to regular consumption of legumes typically included in vegan diets, adequate-protein intake must be expected. It has been established that a plant-based diet normally meets or exceed the recommended protein consumption [1]. These results may explain the similarities in fat-free mass between groups assessed through BIA and we hypothesized no negative impact on body composition associated with protein consumption. Supporting our findings, Nadimi and colleagues [17] performed a similar case-control study between vegetarians and non-vegetarians and evaluated body composition through BIA, 
authors did not identify variations in fat-free mass nor fat mas among participants. Nonetheless, studies which did not contemplate a paired case-control design had concluded that vegetarians present a lower prevalence of overweight according to BMI, and less abdominal and body fat estimated through BIA [7]. Furthermore, the lower protein consumption observed in the vegan group may explain the reduced creatinine concentrations found among these participants $(p=0.010)$ [18]. However, further research regarding this matter is needed to asses this hypothesis and its possible health benefits, such as the improvement in renal function [19].

Even without statistical significance in carbohydrates overall consumption between groups, vegans significantly exceed DRI $(p<0.001)$ due to a larger grain-derived products and legumes intake (317.9 \pm 132.1 vs $248.2 \pm 98.9$ grams; vegans and omnivores, respectively) as a replacement of animal foods. Despite no significant differences in HbA1c concentrations, two vegans presented levels of $\geq 5.7 \%$, probably related to the previously-mentioned excessive carbohydrates daily consumption. Other studies have also found an excessive intake of refined carbohydrates as a replacement of dietary protein and fat in vegetarians, which supposes adverse metabolic changes [9]. No individuals with omnivore diet presented glycemic alterations.

Dietary and soluble fiber intake was significantly greater in vegans ( $p=0.009,0.003$; respectively). These result may implicate positive microbiota modulations. A recent review concluded that vegan's gut profile presents reduced abundance of pathobionts and a greater abundance of protective species [20]. Possible health benefits related to fiber intake may be assumed in favor of the plant-based diet sample, yet more research in this specific topic is needed in this particular population.

Only two individuals from the vegan group declared professional dietetic guidance and intramuscular vitamin B12 and iron supplementation. Lower B-complex vitamins and calciferol intake were identified in vegans when compared with controls $(p<0.001,0.006,0.011,<0.001$; respectively). Nine vitamins were below DRI values in the plant-based diet consumers. Omnivores also share deficient consumption of seven of those vitamins. Cobalamin and calciferol dietary consumption were absent among vegans. These findings could predispose this vegan sample to develop health concerns related to vitamin deficiencies, which could eventually higher the risk of pellagra, associated to lack of daily consumption of niacin (especially in older adults); peripheral neuropathy, glossitis or seborrheic dermatitis related with pyridoxine deficiency; megaloblastic anemia, hyperuricemia, hyperhomocysteinemia, or subacute combined degeneration of spinal cord associated with cobalamin deficiency. Our findings also suggest vitamin $\mathrm{B}_{12}$ subnormal serum concentrations in the vegan group when compared with both controls and standard of reference $(p<$ $0.001)(\leq 200 \mathrm{pg} / \mathrm{mL}, 148 \mathrm{pmol} / \mathrm{L})$. A positive association between feeble cobalamin intake and serum depletion was identified ( $r=0.760 ; p<0.001 ; n=24$, subjects combined), and eight vegan participants were diagnosed with cobalamin deficiency after serum determination. No controls presented cobalamin serum depletion.

In contrast, larger folic acid and vitamin E consumption, associated to a higher intake of fruits (350.6 \pm 184.7 vs. $241.4 \pm 92.6$ grams) and vegetables $(192.3 \pm 74.4$ vs. $168.9 \pm 104.1$ grams $)$ were observed in the plant-based group when compared with controls ( $p=0.007,0.020$; respectively).

Regarding minerals, fluoride, iodine, and selenium daily consumption in vegans were significantly lower than controls ( $p=0.047,0.012,<0.001$; respectively), but higher intake of copper and molybdenum were observed in the plant-based diet group $(p=0.032 ; 0.009)$. Furthermore, vegandiet consumers presented six mineral deficiencies when compared with DRI recommendations (calcium, chromium, fluoride, iodine, potassium, and selenium). Omnivores also presented inadequate ingestion of calcium, chromium, fluoride, iodine, iron, magnesium, potassium, and zinc.

No significant differences were observed between groups for complete blood count analysis. However, macrocitosis (mean corpuscular volume $>97 \mathrm{fL}$ ) and microcitosis (mean corpuscular volume $<97 \mathrm{fL}$ ) were identified in two and four vegan participants, respectively. Additionally, two vegan females were diagnosed with microcytic anemia ( $<12 \mathrm{~g} / \mathrm{dL}$ in women). Based on these findings, we could address non-heme iron intake together with menstruation's cyclic blood loss in female 
subjects as possible origins of anemia. It should be of notice that, although vegans iron intake was superior when compared with controls, the low bioavailability of non-heme iron contained in vegetable food could explain these suggestive findings on iron-deficiency anemia [21]. The abovementioned may also indicate that adult vegan females present higher risk of developing hematologic repercussions, and closer medical-nutritional counseling in this potentially vulnerable group may be addressed. Other studies had also shown that vegetarians have a high prevalence of iron depletion and associated iron deficiency anemia when compared to non-vegetarians, authors also address importance to premenopausal vegetarian women's iron status [22].

Additionally, selenium deficiency in the vegan group may implicate an inadequate activity of glutathione peroxidase enzyme and other selenoproteins, with potential repercussions on polymorphonuclear cells' competency and chronic oxidative stress, respectively [23,24].

Despite these seemingly adverse health outcomes, other studies suggest a lower incidence of chronic diseases in vegans. This could partially result from a beneficial impact of the dietary fatty acid composition. Our group has reported a serum decrease in pro-inflammatory fatty acids suggestive of protective vascular effect on individuals with a vegan diet [25]. Additionally, regular practice of physical activity, management of stress through meditation techniques, and the avoidance of alcohol and tobacco that accompanies the vegan diet in other communities could generate positive results [26]. However, we did not entirely identify those particular health-related behaviors in our sample.

This study provides pertinent evidence to the Mexican population regarding the relationship between a local vegan diet and its health implications. Our results coincide partially with what has been reported in the international literature. This reinforces the hypothesis that an unbalanced vegan diet implies potential nutrients deficiencies, especially vitamin $B_{12}$. However, we strongly suggest future research emphasis on Mexican plant-based diets. Specific nutrients interactions may be explored, as daily intake of tryptophan (precursor of niacin) could diminish $\mathrm{B}_{3}$ vitamin deficiencies' health consequences in the vegan group. Pharmacological interactions when following a plant-based diet must also be analyzed. Vegans could be vulnerable to medications that negatively-interfere with pyridoxine metabolism, such as Isoniazid [27]. It is also pertinent to include homocysteine, holotranscobalamin, and methyl-malonic acid as additional biochemical screening addressing cobalamin deficiency in this vegan community [28-30].

We found an insufficient consumption of several micronutrients in vegans and omnivores. These findings suggest that the local diet may be unbalanced, affecting both dietary groups. Nevertheless, individuals following the local plant-based diet might present additional deficiencies. It is well documented that vegan diets lead to health advantages [2]; however, sociocultural and food sources around the vegan diet in this Mexican population could limit the benefits reported in other latitudes, and clinical and nutritional guidance is required to avoid specific deficiencies with potential health adverse events. Our preliminary results are remarkably valuable for helping provide correct information on appropriate diet planning to subjects who want to initiate with a vegan dietary pattern.

We recognize sample size with unequal numbers of male and female participants as a limitation. Nevertheless, it is considered appropriate that a minimum of twelve subjects per group be included for pilot studies -such as the present research- based on the feasibility and precision around the estimates to be used to design future studies [31,32]. Furthermore, our anthropometric, dietetic and biochemical findings together with a holistic interpretation in this unexplored vegan population are relevant to the international literature and valuable for clinical practitioners, which promotes further larger-research regarding this matter. Future investigations may also consider a prospective randomized trial design to diminish the expected bias of the transversal and observational nature of our study. 


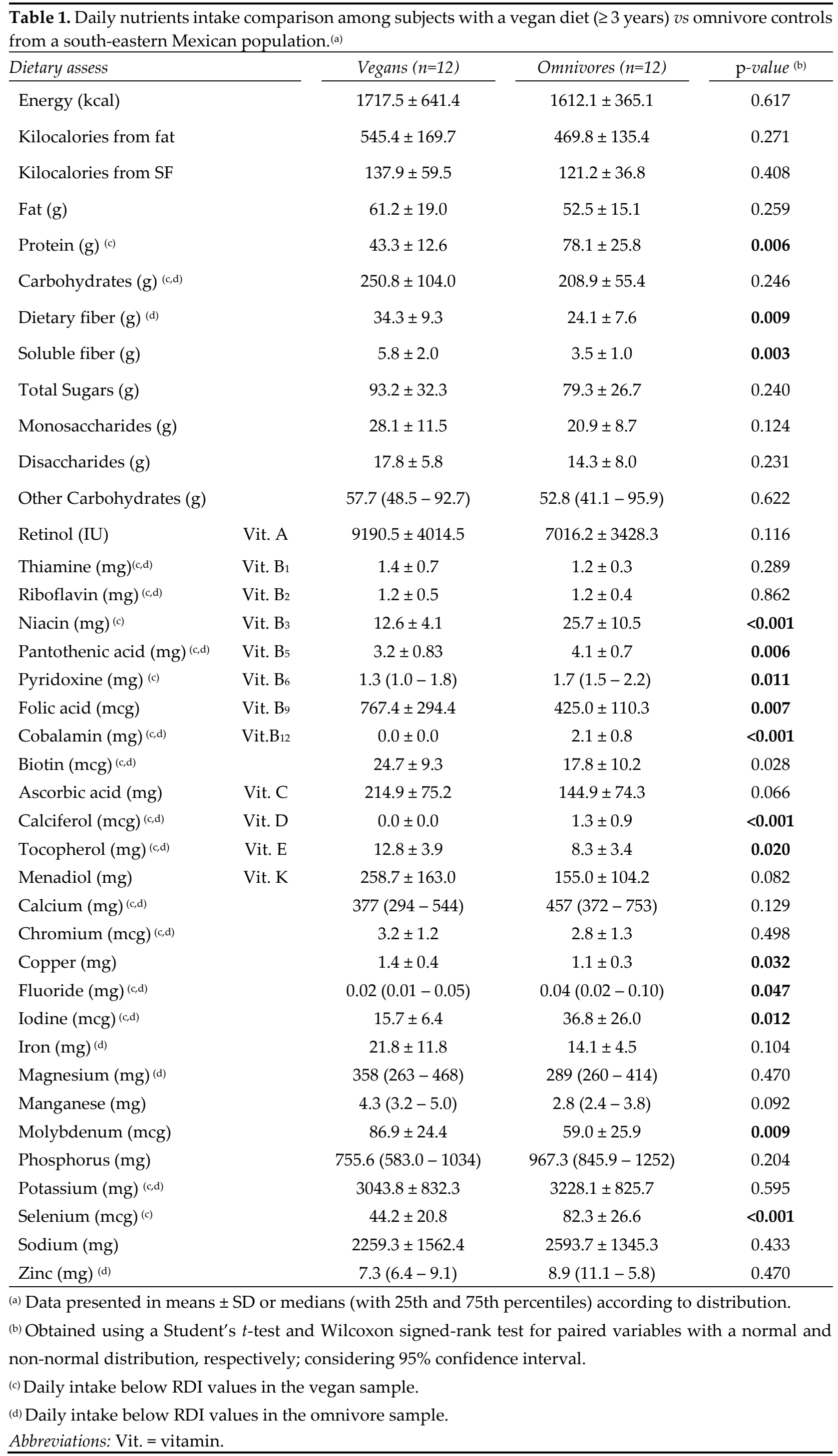


Table 2. Biochemical markers comparison among subjects with a vegan diet ( $\geq 3$ years) vs omnivore controls from a south-eastern Mexican population.(a)

\begin{tabular}{|c|c|c|c|}
\hline Plasmatic biomarkers & Vegans $(n=12)$ & Omnivores $(n=12)$ & $\mathrm{p}$-value $(\mathrm{b})$ \\
\hline $\mathrm{Hb}(\mathrm{g} / \mathrm{dL})$ & $13.4 \pm 1.7$ & $13.5 \pm 0.5$ & 0.907 \\
\hline Hct $(\%)$ & $40.0 \pm 4.1$ & $40.0 \pm 1.2$ & 0.951 \\
\hline MCV (fL) & $89.3 \pm 7.3$ & $87.9 \pm 2.5$ & 0.578 \\
\hline $\mathrm{MCH}(\mathrm{pg})$ & $31.0(28.3-32.0)$ & $30.0(29.0-30.8)$ & 0.699 \\
\hline $\mathrm{MCHC}(\%)$ & $34.0(33.0-34.0)$ & $34.0(33.2-34.0)$ & 0.563 \\
\hline Erythrocytes (cell/ $/ \mathrm{mm}^{3}$ ) & $4,496,667 \pm 488,826$ & $4,551,667 \pm 184,087$ & 0.694 \\
\hline $\operatorname{HbA1c}(\%)$ & $5.2 \pm 0.4$ & $5.0 \pm 0.2$ & 0.271 \\
\hline Vit. $B_{12}(p g / d L)$ & $206.5 \pm 153.4$ & $1560.4 \pm 720.6$ & $<0.001$ \\
\hline Creatinine (mg/dL) & $0.79 \pm 0.13$ & $0.94 \pm 0.16$ & 0.010 \\
\hline
\end{tabular}

(a) Data presented in means \pm SD or medians (with 25th and 75th percentiles) according to distribution.

(b) Obtained using a Student's $t$-test and Wilcoxon signed-rank test for paired variables with a normal and non-normal distribution, respectively; considering 95\% confidence interval.

Abbreviations: $\mathrm{Hb}=$ hemoglobin; $\mathrm{Hct}=$ hematocrit; $\mathrm{MVC}=$ mean corpuscular volume;

$\mathrm{MCH}=$ mean corpuscular hemoglobin; $\mathrm{MCHC}=$ mean corpuscular hemoglobin concentration;

Vit.= vitamin.

International System of Units conversions: $1 \mathrm{~g} / \mathrm{dL}$ of $\mathrm{Hb}$ is equivalent to $0.62 \mathrm{mmol} / \mathrm{L}, 1 \mathrm{pg}$ of $\mathrm{MCH}$ is equivalent to $0.062 \mathrm{fmol}, 1 \mathrm{pg} / \mathrm{mL}$ of Vitamin $B_{12}$ is equivalent to $0.74 \mathrm{pmol} / \mathrm{L}, 1 \mathrm{mg} / \mathrm{dL}$ of Creatinine is equivalent to $88.4 \mu \mathrm{mol} / \mathrm{L}$. 
Acknowledgments: All authors are grateful with Universidad Marista, National Institute of Nutrition "Salvador Zubirán" and the Chemistry Faculty from Universidad Autónoma de Yucatán for the facilities in the usage of the departments, scientific equipment, and software for developing the present investigation.

Conflicts of Interest: The authors declare no conflict of interest.

Funding statement: This work was funded by Universidad Autónoma de Yucatán Chemistry faculty by grant “SISTPROY FQUI-2016-0005" 


\section{References}

1. Melina V, Craig W, Levin S. Position of the Academy of Nutrition and Dietetics: Vegetarian Diets. J Acad Nutr Diet 2016;116:1970-80. doi:10.1016/j.jand.2016.09.025.

2. Dinu M, Abbate R, Gensini GF, Casini A, Sofi F. Vegetarian, vegan diets and multiple health outcomes: A systematic review with meta-analysis of observational studies. Crit Rev Food Sci Nutr 2017;57:3640-9. doi:10.1080/10408398.2016.1138447.

3. Rizzo G, Laganà AS, Rapisarda AMC, La Ferrera GMG, Buscema M, Rossetti P, et al. Vitamin B12 among vegetarians: Status, assessment and supplementation. Nutrients 2016;8:1-23. doi:10.3390/nu8120767.

4. Haider LM, Schwingshackl L, Hoffmann G, Ekmekcioglu C. The effect of vegetarian diets on iron status in adults: A systematic review and meta-analysis. Crit Rev Food Sci Nutr 2016:1-16. doi:10.1080/10408398.2016.1259210.

5. Baumgartner RN, Heymsfield SB, Roche AF. Human Body Composition and the Epidemiology of Chronic Disease. Obes Res 1995;3:73-95. doi:10.1002/j.1550-8528.1995.tb00124.x.

6. Huang RY, Huang CC, Hu FB, Chavarro JE. Vegetarian Diets and Weight Reduction: a Meta-Analysis of Randomized Controlled Trials. J Gen Intern Med 2016;31:109-16. doi:10.1007/s11606-015-3390-7.

7. Acosta Navarro JC, Midori Oki A, Gomes de Gouveia LA, Hong V, Bonfim MC, Cardenas PA, et al. Healthier Body Composition in Vegetarian Men Compared to Omnivorous Men. J Nutr Food Sci 2016;06. doi:10.4172/2155-9600.1000529.

8. Ma C, Avenell A, Bolland M, Hudson J, Stewart F, Robertson C, et al. Effects of weight loss interventions for adults who are obese on mortality, cardiovascular disease, and cancer: systematic review and meta-analysis. BMJ 2017;359:j4849. doi:10.1136/bmj.j4849.

9. Sun C, Hsu H, Chen S. Vegetarian diet is associated with higher SBP and fasting serum triacylglycerol than omnivorous diet in Taiwanese type 2 diabetic. Diabetes Res Clin Pract 2016;120:S141. doi:10.1016/s01688227(16)31284-0.

10. Khalil SF, Mohktar MS, Ibrahim F. The theory and fundamentals of bioimpedance analysis in clinical status monitoring and diagnosis of diseases. Sensors (Switzerland) 2014;14:10895-928. doi:10.3390/s140610895.

11. International Society for the Advancement of Kinanthropometry. International Standards for Anthropometric Assessment. 2001.

12. Monsalve Álvarez JM, González Zapata LI. Diseño de un cuestionario de frecuencia para evaluar ingesta alimentaria en la Universidad de Antioquia, Colombia. Nutr Hosp 2011;26:1333-44. doi:10.3305/nh.2011.26.6.5267.

13. Nationa Institutes of Health. Nutrient Recommendations: Dietary Reference Intakes (DRI) 2011. https:/ods.od.nih.gov/Health_Information/Dietary_Reference_Intakes.aspx (accessed August 30, 2019).

14. Chan M. Haemoglobin concentrations for the diagnosis of anaemia and assessment of severity. Geneva, Switz World Heal Organ 2011:1-6. doi:2011.

15. Diabetes care. Classification and diagnosis of diabetes: Standards of medical care in Diabetes-2018. Diabetes Care 2018;41:S13-27. doi:10.2337/dc18-S002.

16. Solomon LR. Low Cobalamin Levels as Predictors of Cobalamin Deficiency: Importance of Comorbidities Associated with Increased Oxidative Stress. Am J Med 2016;129:115.e9-115.e16. doi:10.1016/j.amjmed.2015.07.017.

17. Nadimi H, Yousefinejad A, Djazayery A, Hosseini M, Hosseini S. Association of vegan diet with rmr, body composition and oxidative stress. Acta Sci Pol Technol Aliment 2013;12:311-8.

18. Wiwanitkit V. Renal function parameters of Thai vegans compared with non-vegans. Ren Fail 2007;29:219-20. doi:10.1080/08860220601098912.

19. Juraschek SP, Appel LJ, Anderson CAM, Miller ER. Effect of a high-protein diet on kidney function in healthy adults: Results from the omniheart trial. Am J Kidney Dis 2013;61:547-54. doi:10.1053/j.ajkd.2012.10.017.

20. Glick-Bauer M, Yeh MC. The health advantage of a vegan diet: Exploring the gut microbiota connection. Nutrients 2014;6:4822-38. doi:10.3390/nu6114822.

21. Fields H, Ruddy B, Wallace MR, Shah A, Millstine D, Marks L. How to monitor and advise vegans to ensure adequate nutrient intake. J Am Osteopath Assoc 2016;116:96-9. doi:10.7556/jaoa.2016.022. 
22. Pawlak R, Berger J, Hines I. Iron status of vegetarian adults: A review of literature. Am J Lifestyle Med 2016;XX:1-13. doi:10.1177/1559827616682933.

23. Swart R, Schutte AE, van Rooyen JM, Mels CMC. Serum selenium levels, the selenoprotein glutathione peroxidase and vascular protection: The SABPA study. Food Res Int 2018;104:69-76. doi:10.1016/j.foodres.2017.06.054.

24. Zoidis E, Seremelis I, Kontopoulos N, Danezis GP. Selenium-dependent antioxidant enzymes: Actions and properties of selenoproteins. Antioxidants 2018;7:1-26. doi:10.3390/antiox7050066.

25. Espinosa-Marrón A, Laviada-Molina HA, Moreno-Enríquez A, Sosa- IF, Molina-Seguí F, Villaseñor-Espinosa MF, et al. Plasmatic Fatty Acids Chemical Characterization after the Exposure to a Vegan Diet. Preprints 2019:1-12. doi:10.20944/preprints201906.0187.v1.

26. Latha Marupudi M, Rao Gondi S, Sesha Sai Krishna Manne V, Yalavarti S. Confounding Effects of Age, Diet and Physical Activity on Blood Pressure. J Evol Med Dent Sci 2017;5:1888-91. doi:10.14260/jemds/2016/446.

27. Mason P. Important drug-nutrient interactions. Proc Nutr Soc 2010;69:551-7. doi:10.1017/S0029665110001576.

28. Herrmann W, Schorr H, Purschwitz K, Rassoul F, Richter V. Total Homocysteine, Vitamin B12, and Total Antioxidant Status in Vegetarians. Clin Chem 2001;47:1094-101.

29. Krajčovičová-Kudláčková M, Blažíček P, Kopčová J, Béderová A, Babinská K. Homocysteine levels in vegetarians versus omnivores. Ann Nutr Metab 2000;44:135-8. doi:10.1159/000012827.

30. Haddad EH, Berk LS, Kettering JD, Hubbard RW, Peters WR. Dietary intake and biochemical, hematologic, and immune status of vegans compared with nonvegetarians. Am J Clin Nutr 1999;70:586-93. doi:10479236.

31. Julious SA. Sample size of 12 per group rule of thumb for a pilot study. Pharm Stat 2005;4:287-91. doi:10.1002/pst.185.

32. Rogerson D, Maçãs D, Milner M, Liu Y, Klonizakis M. Contrasting effects of short-term mediterranean and vegan diets on microvascular function and cholesterol in younger adults: A comparative pilot study. Nutrients 2018;10. doi:10.3390/nu10121897. 